\title{
Performance Evaluation of Unicast Routing Protocols in MANETs - Current State and Future Prospects
}

\author{
https://doi.org/10.3991/ijim.v11i1.6295 \\ Mohammad Alnabhan \\ Mu'tah University, Mu'tah, Jordan \\ m. alnabhan@mutah.edu.jo \\ Mahmoud Alshuqran \\ Jerash University, Jerash, Jordan \\ shugran67@yahoo.com \\ Mustafa Hammad \\ Mu'tah University, Mu'tah, Jordan \\ hammad@mutah. edu . jo \\ Mohammad Al Nawayseh \\ Jordan University, Amman, Jordan \\ m. nawaiseh@ju.edu.jo
}

\begin{abstract}
Mobile Ad hoc Networks (MANETs) are considered as a reunion of wireless mobile devices (nodes) that form a temporary wireless network. In order to facilitate communication in MANET, every node has to participate in the routing process. Reaching an optimal route is a fundamental task in MANET, because routes are multi-hoped and susceptible. Several routing protocols exist and can be categorized to; topology-based and position-based routing protocols. However, the efficiency of these protocols in highly dynamic and dense environments is a challenging task to be considered for increasing perceived Quality of Service (QoS) in MANET. This paper focuses on the presentation and basic operation of each category. A performance evaluation study was conducted comparing between both categories in terms of End-to End delay, packet- delivery ratio and routing overhead. Results analysis show that position-based protocols outperforms topology-based protocols in dense and high dynamic environments. Recommendations for implementing future efficient position-based protocols were presented.
\end{abstract}

Keywords-MANET, Multi-hop, position-based, Routing, Topology-based. 


\section{Introduction}

Mobile Ad-hoc Networks (MANET) gain more interest with reference to its' potential deployment values [1]. MANETs can extend communication beyond the limit of infrastructure-based networks. The importance of such structure is allocated within areas where infrastructure based communication cannot be achieved. These places include disaster recovery situations and battlefield operations. Furthermore, the usage of MANETs can extend for other places like, conferences, electronic classrooms, and airport area, where users create a network without using pre-existing network [2].

As of its infrastructure and operational environment, MANET gains several challenges. This includes routing, security, power consumption, and quality of service [3]. Among these issues, routing is one of the most fundamental yet challenging problems for MANETs. Hence, new and continuous demands are imposed on the routing protocol design $[2,3]$ and $[4]$.

This paper provides an overview of available unicast routing protocols. Furthermore, it present a qualitative comparison between most known unicast routing protocols with reference to an in depth analysis of QoS variables including End-to-end delay, routing overhead and packet delivery ratio. The remainder of this paper is structured as following. Section 2 presents a short overview of the chosen protocols. Section 3 shows the simulation environment. The results of simulation scenarios are discussed in Section 4. Section 5 presents final remarks and conclusions.

\section{Recent Classification And Comparison Of Manet Routing Protocols}

Many research works have discussed and classified routing issues for MANETs [5, 6] and [7]. An extensive survey focused on proactive, reactive and geographic MANET routing protocols based on efficient flooding techniques was described in [7]. Similar survey was conducted by [8], and a new routing category was introduced and described as power-aware routing protocols, which can be integrated with previous categories. In addition, MANET routing protocols were divided into Reactive, Proactive and Hybrid protocols in $[9,10]$. A detailed comparison and analysis between these three types of topology based routing protocols was discussed, and desirable properties for MANET routing protocols was described in these papers.

A new classification for MANET routing protocols was provided in [11] based on routing strategy. Two main categories were described; table driven and on demand routing protocols. A qualitative analysis for these two categories was conducted to deduct features, differences and characteristics of each category.

In $[12,13]$ and [16] a performance comparative study was conducted between two types of topology-based routing protocols; on-demand routing protocols DSR, AODV and TORA and table driven protocol DSDV. Considering packet delivery, DSR and AODV perform the best independent of the number of sources. However, in high mobility scenarios DSDV packet delivery is very low and its overhead was very high. 
During all scenarios, DSR performance was the best comparing to all other protocols for all metrics.

A similar comparative study was conducted in [14] and [15], a performance comparison between DSR, AODV and DSDV protocols. It was observed that AODV outperforms DSR and DSDV in most scenarios, when number of nodes were above 100. However, DSR performed well in most cases when number of nodes was less and around 100 for the particular scenario. The delay time for using DSR and DSDV increases very rapidly with the increase number of nodes, while AODV experienced delay is consistent with the increasing number of nodes. In case of packet loss, DSR experience was the minimum in all cases while number of nodes were limited, as compared to AODV and DSDV. DSDV was having maximum packet losses in case of varying pause time, simulation time and speed.

A comparative analysis between topology-based and position-based routing protocols was described in [9]. Topology-based routing includes AODV and DSR. Position-based (geographic) routing protocols are GSR (Geographic Source Routing), GPSR (Geographic Position Source Routing), and A-STAR (Anchor-based Street and Traffic Aware Routing). Simulation results have specified that GPSR and GSR have minimal packet delivery ratio using fixed CBR rate. However, during high density environment, GSR outperforms GPSR with reference to packet-delivery ratio. In addition, GSR outperforms topology based protocols (AODV and DSR) with reference to latency and packet delivery ratio. In the other side, GPSR incurred high endto-end delay with increased CBR rates. A-STAR achieves over all enhanced network performance comparing to position-based protocols (GSR and GPSR). Using these results, [9] presents a new position-based routing protocol known as predictive directional greedy routing (PDGR). The objective was to forward packets to suitable next hop using predicable and current future situations.

\section{$3 \quad$ Routing In Manets}

Routing in MANET is a challenge task due to limited resources and rapid changing network topology. Moreover, establishing and maintaining routes in such environment required high control packets which make routing process more challenge. Therefore, numerous routing protocols have been developed with optimized and efficient routing functions to deal with MANET challenges. With reference to utilizing geographic location information, MANET routing protocols are divided into two main types; location-aware (position-based) and location-unaware (Topology-based) routing protocols [4].

\subsection{Topology-based Routing Protocols}

Location-unaware or topology-based routing protocols uses information about network links and connections to perform packet forwarding. Hence, each mobile node has to maintain up-to-date routing tables by continuously exchanging routing 
formation. Topology-based protocols are divided into three categories: reactive, proactive and hybrid protocols [4].

Proactive Routing Protocols: This category is known as table driven routing protocol, where nodes periodically exchange topology information even if this information is not currently used. Although, proactive protocols provide shortest path information, there is no need to have initial route discovery, which decrease end-toend delay. On the other hand, for periodic update of topology, such protocols consume memory and power, increase network overhead, and consume lot of bandwidth. Based on these drawbacks, proactive routing protocols are only suitable to small size networks [10]. Destination-Sequenced Distance-Vector (DSDV) [8] is an example of this category of routing protocols.

Reactive Routing Protocols: In this approach nodes periodically exchange topology information only when there is data to be sent. On other words, the reactive routing protocols establish the route on demand. Compared to proactive protocols, deploying this protocols category reduces network overhead, because it requires less bandwidth, and needs less memory. However, reactive routing protocols suffer from initial route discovery process, thus more end-to-end delay is incurred to establish the route. Compared to the proactive protocols the reactive routing protocols are suitable to medium scale networks [5]. Dynamic source routing (DSR) [7], is an examples of this category of routing protocols.

Hybrid Routing Protocols: Hybrid routing protocols represents an integration between table driven and on-demand routing protocols. Hybrid routing protocols utilize advantages and prevent the side-effects of both categories. Such routing protocols decrease the delay of on-demand routing protocols and reduce overhead for table driven routing protocols. On the other hand, Hybrid routing protocols need to continuously maintain network paths that are currently in use. Accordingly, this will lead to significant control overhead traffic that consumes node's battery power and reduces the bandwidth for application data. Hybrid routing protocols meet the requirements of large scale networks. Zone routing protocol (ZRP) [11], is an examples of this category.

\subsection{Position-Based Routing Protocols}

The second category is described as position-aware or position-based routing protocols. This category protocols operates based geographic positioning information of neighboring nodes to make routing decisions and do not relay on link state information as in topology-based.

Position-based routing aims to make progress toward the destination by forwarding packets from source or forwarder node to a neighbor within its transmission range. This neighbor node is closer to the destination than the forwarder node. Generally, any position-based routing protocols consists of two main operations: the location service protocol and the actual routing protocol of data packets. Predictive Directional Greedy Routing (PDGR) [9], is an examples of this category of routing protocols. 


\section{$4 \quad$ Selected Routing Protocols}

The following section describes routing protocols being selected within the performance comparative study conducted in this work.

\subsection{Destination-Sequenced Distance-Vector Routing (DSDV)}

DSDV is one of the well-known table-driven (proactive) routing protocols. DSDV assure the use of single shortest path to destination with loop freedom [10]. It is proactive because each and every node in the network has full information of available routes to all reachable destinations in advance. Each node maintains an up to date routing table.

This routing table holds information of the next hop for all reachable destinations. Typically, routing table records consist of destination address, latest sequence number received from destination, and sum of hops to reach destination. When a node receives a new rout information, it make a comparison between new and old sequence numbers stored in the routing table. Afterwards, rout with greatest sequence number will be kept and the other is discarded [12].

In case both sequence numbers are the same, then the one with lower number of hops is used. Thus, the node is prevented to forward data with the same sequence number twice to avoid loops. Moreover route should be labeled with the latest sequence number to avoid stale routes.

The routing table in each node is updated whenever new information is available or in periodically manner. For the latest method, the periodic update interval value determines routing protocol performance. If the interval value is very small, then the routing overhead will be large [10]. In addition, if interval value is very long, then there will be a delay to get the fresh route information.

\subsection{Dynamic Source Routing (DSR)}

DSR presented in [7], is a reactive (on demand) routing protocol that utilize shortest hop path between source and destination. As the name indicates, DSR is a source routing protocol, where the sender determines the entire path to the ultimate destination.

With DSR, nodes maintain a route cache; each entry in the route cache holds complete routs to a final destined node. When a node requires sending data packet to a certain destination, the rout is determined by searching its cache or by conducting a route discovery process. Hereafter, it inserts the collected information about the path address in the packet header [12].

In case the rout to destination is not available in the cache, a node initiates and broadcasts a route request packet (RREQ). The RREQ holds a path record in which the sequence of next hop information is saved along with a unique request ID. Along the route, each and every intermediate node receives the route request packet. If any node holds corresponding entry in its cache for ultimate destination node, it will send a route reply (RREP) message to the initiator or source node. If there was no entry in 
intermediate node's cache, it forwards the packet after adding its address. This is repeated until RREQ reaches to destination. In turn, the destination uses the revers path to send RREP to source [10].

\subsection{Zone Routing Protocol (ZRP)}

ZRP is a hybrid routing protocol proposed by [11]. This protocol integrates on demand and table driven routing protocols. ZRP prevents drawbacks of reactive and proactive protocols. Thus, it produces less overhead compared to proactive protocols and less delay compared to reactive protocols, therefore, ZRP is suitable for large networks.

Using ZRP, network nodes are grouped in small parts called "routing zones". Each node should belong to one zone based on its geographical position. In ZRP, the zone radius is a critical metric determined by the number of hops, thus it should be selected carefully, and otherwise a participating node can be a member in multiple zones [11].

Whenever a source node wants to communicate with specific destination, it proactively looks for destination within its zone, if it does not exist; source node reactively sends a route request packet to neighboring zones. Any node receiving PREP and knowing the rout to destination, sends a route reply to the initiator node, otherwise, address is added and the request is rebroadcasted until it reaches to ultimate target [5, 8].

\subsection{Predictive Directional Greedy Routing (PDGR).}

PDGR was presented in [9]. This protocol considers both moving direction and location of each node, and the next-relay hop should be the closest to destination. PDGR integrates Direction First Forwarding (DFF) and Position First Forwarding (PFF). Thus, the weighted score is computed from these two strategies. The weighted score is computed for the source and its current neighbors, and prospective neighbors. This routing information is obtained using beacon messages. PDGR decisions are used to select next-relay hop for packets forwarding, this is achieved using predicted mobility information in the current and future state. If information about the neighbor is unavailable, using higher weighted route score, the source node forwards the packet to reach its neighbor.

\section{Simulation Study}

The Performance comparative study between selected position-based and topology-based protocols is conducted using a simulation model implementing these protocols in real scenarios. 


\subsection{Simulation Set-up}

Routing protocols performance was measured using Network Simulator 2 (NS2) [18]. Default specifications of routing protocols were considered. Transmission range was $250 \mathrm{~m}, 15$ source and destination pairs were chosen randomly. The simulation network space was $2500 \mathrm{~m}$ x $2000 \mathrm{~m}$. In addition, 802.11 DCF RTS/CTS was used as the MAC layer protocol. Bandwidth, queue size and data packet size were set to standard values; 2 mbps, 50 packets, and 512 byte respectively. Beacon packet size was 64 bytes. Continuous Bit Rate (CBR) traffic model was used with data rated suited to 5 packets/s. Each simulation scenario lasted for 1200 seconds, only data measured between $800 \mathrm{~s}-1000$ s was considered to neglect the effect of initial setup and ending states.

Experiments were conducted using two different scenarios. The first scenario implements a mobile environment with different nodes' density moving with fixed speed. In this scenario, the number of mobile nodes considered; 50, 100, 200, 300, 400 , and 500 , with fixed speed of $20 \mathrm{~m} / \mathrm{s}$. The second scenario used a fixed number of nodes with different mobile nodes' speed. Speed values considered in the second scenario were; 40, 30, 20, 10, and $5 \mathrm{~m} / \mathrm{s}$, and the number of nodes was fixed to 200 . An average of 10 different simulation trials was conducted.

\subsection{Set-up of Mobility Model-Random Waypoint}

Simulation parameters with reference to Random Waypoint Mobility Model are described in table 1 . Packet initially travels from a random location to a random destination using arbitrarily selected speed. When destination is reached, nodes move to a further randomly chosen destination. Duration pause time affects relative speeds of mobile nodes, where speed is set to zero to be compatible with other routing protocols at the same time.

Table 1. Simulation Parameters Of Random Waypoint Mobility Model

\begin{tabular}{|l|l|l|}
\hline Description & Value & Unit \\
\hline Pause time & 0 & $\mathrm{~s}$ \\
\hline Speed interval & {$[1,40]$} & $\mathrm{m} / \mathrm{s}$ \\
\hline Motion direction & {$[0,2 \pi]$} & degree \\
\hline
\end{tabular}

\subsection{Performance Evaluation variables}

During simulation, the following performance metrics were considered:

- End-To-End Delay (E-2-E): This variable describes the difference between data packet generation time at source $\boldsymbol{T s}$, and reception time at destination $\boldsymbol{T d}$.

- Packet Delivery Ratio (PDR): This variable represents the ratio between the number of data packets received successfully at the destination, to the number of packets being sent.

- Routing Overhead Ratio: This variable presents the ratio between transmitted control packets to each received data packet. 


\section{Experimental Results}

\subsection{First Scenario: Various Numbers of Nodes}

In the first scenario, a variance number of participating nodes was used and nodes' moves in a static speed value equal to $20 \mathrm{~m} / \mathrm{s}$ and. Using these settings, Figure $1 \mathrm{de}-$ scribes results of evaluating routing protocols based on the Packet delivery ratio (PDR), End-to-end (E-2-E) delay, and Routing Overhead.

Figure 1.a describes PDR values within different participating nodes. Very similar values were achieved for topology-based protocols ZRP, DSR, whereas DSDV has the lowest value. In this concern, position-based protocol PDGR outperforms all protocols by achieving PDR values ranging from $(60-90) \%$ considering variant number of nodes.

In addition, PDGR outperforms topology-based protocols with reference to routing overheads and average end-to-end delay by achieving lowest values with increased number of nodes; see figures $1 \mathrm{~b}$, and $1 \mathrm{c}$. An average of 2 seconds end-to-end delay was achieved by PDGR while number of nodes ranged from $(50-500)$. However, the best average delay attained by topology based protocols was 4 seconds within same settings.

For routing overhead the worst and fluctuated performance of the three topology protocols is realized by the proactive DSR protocol, see Figure 1c. In which, the increased number of nodes has dramatically increased the number of control packets, reaching to 3000 packets at 500 nodes' number size.

To sum up, results confirm that topology-based protocols experience difficulties to scale in dens networks with more than few hundred of nodes.

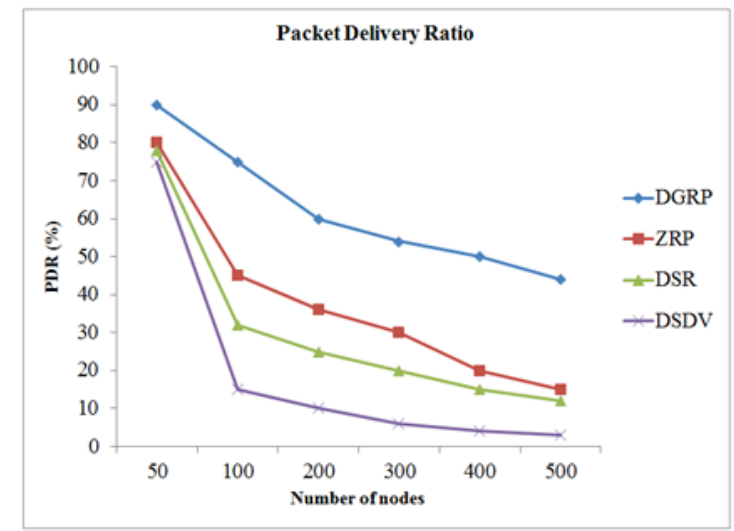

a) Packet delivery measurements for different number of nodes 


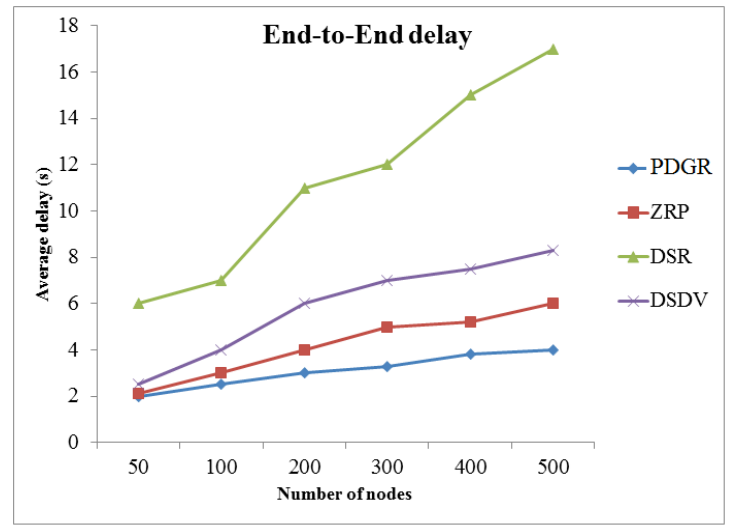

b) End-to-End Delay measurements for different number of nodes

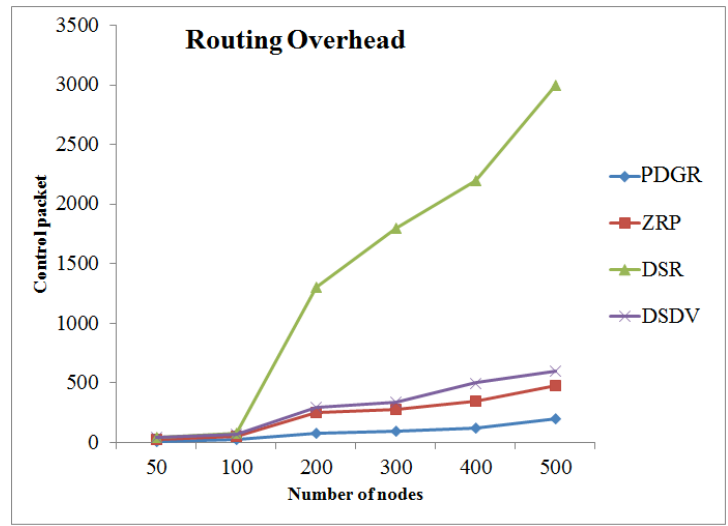

c) Routing Overhead for different number of nodes

Fig. 1.

\subsection{Second Scenario: Different Node Mobility}

This scenario utilizes a fixed number of 200 participating nodes, and a variety movement speeds; 40, 30, 20, 10 and $5 \mathrm{~m} / \mathrm{s}$ were deployed. Using these settings, the impact of mobility on selected routing protocols was measured taking into consideration performance metrics described in section $\mathrm{V}$.

As described in Figure 2a, PDR is not highly affected by low nodes' speed, especially if speed is lower than $10 \mathrm{~m} / \mathrm{s}$. ZRP and DSDV protocols has nearly identical values of PDR at low speed rates and both outperform DSR protocol. While nodes speed increase PDR decreases. A significant drop of PDR was noticed by topologybased protocols when speed higher than $10(\mathrm{~m} / \mathrm{s})$. However, PDGR protocol achieved highest PDR values and shows a steady performance comparing to topology-based routing protocols, especially when speed reaches $20 \mathrm{~m} / \mathrm{s}$. 
For all protocols E-to-E delay tends to be stable as the speed increases. As shown in Figure 2b, ZRP outperforms both DSDV and DSR protocols with a maximum delay of $3 \mathrm{~s}$ when speed reaches $40 \mathrm{~m} / \mathrm{s}$. At the same time, PDGR outperforms topology-based routing protocols with an average delay of $2 \mathrm{~s}$ during variant nodes mobility speeds.

During high mobility, DSR causes high control packet updates, reaching to 1600 packets when mobile speed increases to $40 \mathrm{~m} / \mathrm{s}$. PDGR scheme does not employ updating messages and decrease overhead amount saving network bandwidth, see Figure 2c. Hence, PDGR outperforms topology-based routing protocols in terms of network overheads, where the maximum number of control packets being incurred by this protocol was 50 packets when node speed reaches $40 \mathrm{~m} / \mathrm{s}$. Accordingly, the degree of mobility degrade the performance of all routing protocols.

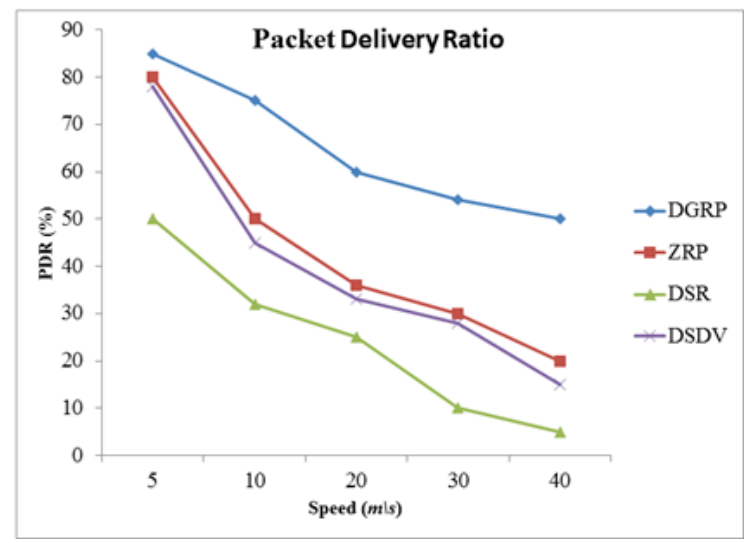

a) Packet delivery measurements for different mobile nodes/ speeds

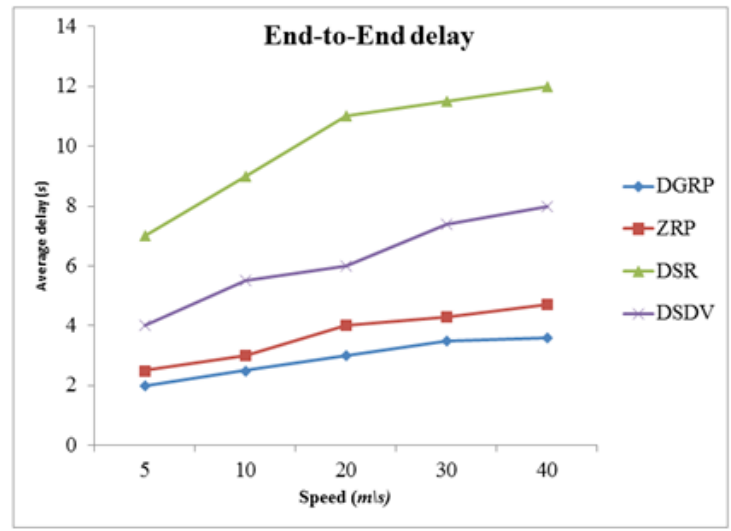

b) End-to-End Delay for different mobile nodes' speeds 


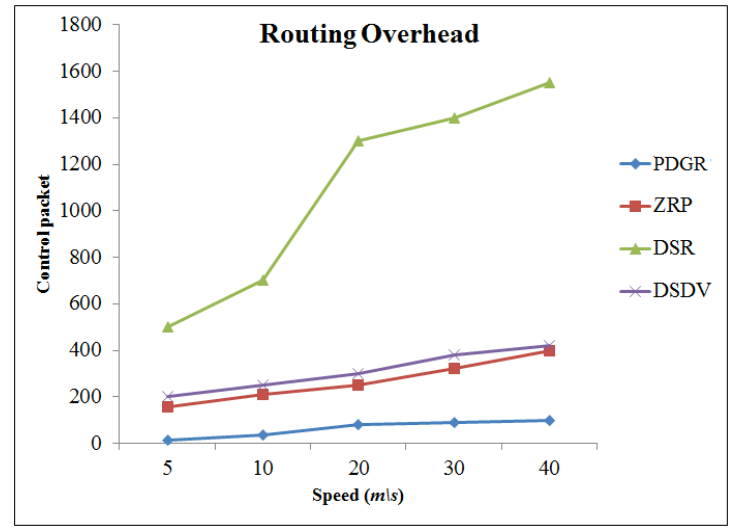

c) Routing Overhead for different mobile nodes' speeds

Fig. 2.

\section{$7 \quad$ Results Analysis and Discussion}

The increased overhead for topology-based routing protocols as described in figures ( $1 \mathrm{~b}$ and $2 \mathrm{~b}$ ), was due to flooding and distribution of network topology information and frequent updates used by these protocols to maintain up-to-date routing lists. This results in significant control traffic that consumes node's power and bandwidth. On contrary, maintenance of routes is not required by position-based routing protocols as in traditional proactive routing protocols, so it prevent extra overhead to be occurred, also it prevent increased latency of rout discovery. In addition, communication overhead in position-based routing is relatively small. Whereas mobile nodes have neither to store routing lists or transmits messages to update routing lists.

In topology-based protocols route maintenance is initiated after link-breakage takes place, causing packet lost and increased delay for new path establishment. This justifies low packet delivery ratios and increased delay achieved by topology-based protocols during experiments, as shown in figures (1a,b and $2 a, b)$. However, with positionbased routing, as the link breakage occurs, forwarding process allows a packet to adapt to topology changes by selecting next best choice. Hence, packets do not have to be dropped.

In addition, position-based routing eliminates the need for route setup time, because data and control packets are transmitted to known coordinates of destination node. Hence, low routing overhead was incurred using PDGR as shown in figures 1c and $2 \mathrm{c}$. This is considered as an important advantage when network topology is continuously changing, as in MANETs environments.

Comparing to previous research, performance results achieved in this work agrees with results described in [9]. A steady and high performance routing was recorded using position-based protocols especially in high dense and dynamic mobile environments. With regard to topology-based routing protocols comparative results achieved 
in this work contradicts results recorded in [10], more precisely in high mobility scenarios. However, in terms of PDR variable, DSR performance recorded in [12] was similar to performance achieved in this work within low network density.

Accordingly, considering geographic routing features and results discussed earlier, it is more appropriate to apply position-based protocols for MANETs rather than topology-based protocol, especially in increased mobility and network size. Positionbased routing ensures scalable and robustness solution for routing in highly dynamic and dense wireless environments. The only constraint of this approach is the limited availability of accurate location information required for geographical routing and packets delivery.

The proposed performance analysis in this work has covered all possible MANETs' scenarios and achieved results were compared and validated with results achieved by previous researches. In addition, this work has recorded the performance of hybrid routing protocol ZRP, which outperforms other topology-based protocols DSDV and DSR. Hence, this has allowed drawing attention to vital recommendations for the design and development of new position-based routing protocols.

Generally, intended protocol should find best path with guaranteed limited end-toend delay and reduced control overhead traffic. Also, low percentage of data loss should be ensured. In general, the following features should be considered:

- A cross-layer, simple standalone and adaptive to unpredictable MANET environment conditions. Therefore, the intended protocol is energy and congestion adaptive, and has the ability to efficiently employ the neighbors' degree.

- Support scalability and handles increased node mobility and network size. It should operate with minimal control overheads, loop freedom and avoid timeout problem.

- The accuracy of participating nodes' location is a critical issue to be considered. In addition, ensuring the integrity of location information contained in nodes neighbors-list is very important, especially in high nodes' mobility. Hence, the intended protocol should have a proper mechanism to remove stale information from the neighbors-list and provide reliable beaconing updates. It is important to adopt localization algorithm able to accurately identify updated mobile nodes location.

- The ability to construct a reliable (optimal) route between communicating nodes considering highest reminder energy, least congestion, and highest connectivity degree nodes.

\section{Conclusion}

This work has investigated MANETs topology-based and position-based protocols taking into consideration methods of operation, weaknesses, strength and performance. An intensive evaluation study was conducted analyzing protocols' performance in different mobility scenarios and settings. Scenarios include several levels of dense and dynamic networks, and covered performance validation factors such as; Packet Delivery Rate (PDR), End-to-End Delay and Routing Over Head. 
Two main experimental settings were considered simulating MANETs operational environment; the first setting considers effects of nodes number size, and the second focuses on variant nodes mobility speeds. Three topology-based protocols were investigated known as; DSR, DSDV and ZPR, and one position-based protocol were considered known as PDGR. Results have confirmed the efficiency of position-based protocols in dynamic and dense environments, where PDGR protocol showed an increased average of PDR within high nodes speed and increased number size. In addition, while running both experiments PDGR incurred less delay and requires low control overheads comparing to topology-based protocols under study.

\section{References}

1. I. Chlamtac, M. Conti, J. Liu, "Mobile Ad Hoc Networking: Imperatives And Challenges," Ad Hoc Networks, vol. 1, No. 1, pp. 13-64, 2003. https://doi.org/10.1016/S1570$\underline{\text { 8705(03)00013-1 }}$

2. V.B. Narsimha, B. Sujatha, and T. SampathKumer "A Survey of Wireless Mobile Ad-hoc Networks (MANET)," International Journal of Science and Advanced Technology, Vol. 1, No 5, pp. 189-191, 2011.

3. A. Boukerche, B. Turgut, N. Aydin, M. Ahmad, L. Boloni, D. Turgut. "Routing Protocols in Ad Hoc Networks: A Survey," Computer Networks (Elsevier), vol.55. No.13, pp.30323088. 2011. https://doi.org/10.1016/j.comnet.2011.05.010

4. D. Grover and S. Saini "A Survey on Unicast Routing Protocols in Mobile Ad-Hoc Networks", International Journal of Advanced Research in Computer Science and Software Engineering Research, vol.5, Issue 5, 2015.

5. S. Pathak and S. Jain "A Survey: On Unicast Routing Protocols for Mobile Ad Hoc Network", International Journal of Emerging Technology and Advanced Engineering, Volume 3, Issue 1, 2013.

6. S.S. Dhenakaran and A. Parvathavarthini, "An Overview of Routing Protocols in Mobile Ad-Hoc Network", International Journal of Advanced Research in Computer Science and Software Engineering, 2013.

7. Maltz, David B. Johnson David A., and Josh Broch. "DSR: The dynamic source routing protocol for multi-hop wireless ad hoc networks." Computer Science Department Carnegie Mellon University Pittsburgh, PA (2001): 15213-3891.

8. C. Perkins, P. Bhagwat, "Highly Dynamic Destination-Sequenced Distance-Vector Routing (DSDV) for mobile computers" in: ACM SIGCOMM, August-September 1994, pp. 234-244.

9. J. Gong, et al, "Predictive Directional Greedy Routing in Vehicular Ad hoc Networks," In Proceedings of International Conference on Distributed Computing Systems Workshops (ICDCSW), pp. 2-10, Jun. 2007. https://doi.org/10.1109/ICDCSW.2007.65

10. Y. H. Jazyah, and H. Martin "A review of routing protocols for UWB MANETs." Computational Science and Its Applications-ICCSA 2010. Springer Berlin Heidelberg, 2010. 228-245. https://doi.org/10.1007/978-3-642-12179-1 21

11. Z. J. Haas, M. R. Pearlman, and P. Samar, "The Zone Routing Protocol (ZRP) for Ad-hoc Networks," in Internet Draft, draft-ietf-manet-zonezrp-04.txt, July 2002.

12. H. Ehsan, and A. U. Zartash "Performance comparison of ad hoc wireless network routing protocols." Multitopic Conference, 2004. Proceedings of INMIC 2004. 8th International. IEEE, 2004. 
13. M. Al-Shugran, et al. "A qualitative comparison evaluation of the greedy forwarding strategies in Mobile Ad Hoc Network." Journal of Network and Computer Applications 36.2 (2013): 887-897. https://doi.org/10.1016/j.jnca.2012.10.008

14. S.S. Tyagi R.K. Chauhan, "Performance Analysis of Proactive and Reactive Routing Protocols for Ad hoc Networks", 2010, International Journal of Computer Applications, Volume 1, No.14, pp.0975-8887.

15. S. Ravikanti and G. Preeti "Evaluating the Performance of Reactive Unicast Routing Protocols with OPNET Simulator in MANETS under VOIP”, International Journal of Innovative Research in Science, Engineering and Technology, Vol. 4, No.7, 2015.

16. O.O Omitola "Performance Evaluation of Routing Protocols in MANETs using Varying Number of Nodes and Different Metrics". African Journal of Computing \& ICT, vol 8. No.2. 2015.

17. B. Chandra, M. Astha Puri, S. Latifi "Performance Assessment of MANET Routing Protocols", International Journal in Communications, Network and System Sciences, vol.8, pp.456-470, 2015. https://doi.org/10.4236/ijcns.2015.811041

18. "The Network Simulator - ns-2," http://www.isi.edu/nsnam/ns/, 2002.

\section{Authors}

Mohammad Alnabhan is an associate professor at Information Technology Department, Mu'tah University, Mu'tah 61710, Jordan.m.alnabhan@mutah.edu.jo.

Mahmoud Alshuqran is an assistance professor at Computer Networks Department, Jerash University, Jerash 26150, Jordan. shugran67@yahoo.com.

Mustafa Hammad is an associate professor at Information Technology Department, Mu’tah University, Mu’tah 61710, Jordan. hammad@mutah.edu.jo

Mohammad Al-Nawayseh is an assistance professor at Management Information Systems Dept., Jordan University, Amman 11942, Jordan. m.nawaiseh@ju.edu.jo

Submitted 24 September 2016. Published as resubmitted by the authors 22 December 2016. 